EPJ Web of Conferences 32, 04018 (2012)

DOI: 10.1051/epjconf/20123204018

(C) Owned by the authors, published by EDP Sciences, 2012

\title{
Electromagnetic modelling of dielectric loaded aperiodic gyrotron beam tunnels
}

\author{
J.G. Chelis ${ }^{1}$, and J.L. Vomvoridis ${ }^{1}$ \\ ${ }^{1}$ National Technical University of Athens, School of Electrical and Computer Engineering, \\ Association EURATOM- Hellenic Republic, GR-15773, Greece
}

\begin{abstract}
We present a new modelling approach for the dielectric loaded gyrotron beam tunnel. We treat the problem realistically as a finite sized structure by considering for the first time open boundary conditions. Matching the field of discrete spectrum in the rings, with the field of continuous spectrum in the hollow region, produces Fourier integrals that are evaluated by use of contour integration and residue theory. The final linear system of equations for the mode coefficients is numerically solved. The method can be extended for finding the complex eigenfrequencies of the structure as well as for modelling more advanced concepts of beam tunnels.
\end{abstract}

\section{Introduction}

The gyrotron beam tunnel is a structure prior to the cavity that is susceptible to the excitation of parasitic oscillations. These parasitic modes interact with the electron beam and alter its properties causing a reduced efficiency of the principal interaction in the cavity. They cause also additional heating that can cause serious damage to the structure.

In order to suppress these parasitics, one typically loads the structure with a lossy dielectric as a means to decrease the quality factor and prevent the excitation of parasitic oscillations. However this scheme has failed in some cases and therefore modified geometries of beam tunnels have been used. These include the introduction of azimuthal indentations on the metal rings or the use of a continuous dielectric structure without metal rings. For the time, these modified geometries seem to be successful in suppressing the parasitic oscillations but as high power gyrotrons with higher beam currents are needed, the problem may become crucial in the future. For that reason efficient and reliable simulations that will ensure the beam tunnel's performance are needed.

Simulating a complex electromagnetic structure such as the beam tunnel is not an easy task. In particular, commercial electromagnetic numerical packages are too slow and they need a large amount of computational resources because the structure is highly overmoded. Furthermore the details and physical insight of such simulations are not always transparent and they cannot help the design in this regard.

On the other hand existing semi-analytic codes (e.g. Fishbone $[1,2])$ have to make considerable simplifications because the geometry is too complicated to model realistically. The major simplification is the assumption of a periodic continuation of the structure that allows the formulation of the problem by Floquet's theorem and space harmonics method. The periodic boundary conditions impose errors as the number of periods of the real structure is not too large. They also impose limitations on further realistic modelling of the structure. For example a varying 
magnetostatic field that typically exists in the beam tunnel cannot be modelled, as it has to maintain the same magnitude at the borders of each period. A further simplification that is usually made is neglecting the recess region that protects the dielectrics from charging by escaped electrons. The recess should play a role for the formation of the field profile in the hollow region and consequently for the interaction with the electron beam. A final serious simplification is the assumption of a constant radius along the structure. As all beam tunnel structures have a decreasing radius, the latter simplification is also a source of errors.

In this work we are formulating the problem on a new basis. Instead of the periodic boundary conditions we apply open boundary conditions without reflections at the ends. This is by far a more realistic approach that allows for further enhancements in the model. By considering also the recess region the model becomes more reliable and resembles the real geometry. In our model we still keep the assumption of constant radius, an assumption that can be revised in the future. The modelling is performed by analytic means that give physical insight to the problem and make also efficient use of computational resources.

\section{Electromagnetic modelling}

\subsection{Geometry}

The geometry of the structure modelled, is depicted in Figure 1. A beam tunnel section consists of a number of dielectric rings stacked between metal rings. In this work we neglect the tapering of the inner radius of the hollow section and as a first simplification we consider this radius $r_{i n}$ constant. An arbitrary number of dielectric rings can be modelled, each with different geometric or physical characteristics such as width $d_{i}$, outer radius $r_{i}$ or relative permittivity $\varepsilon_{\mathrm{r} i}$. Each dielectric ring has also a recess region between the inner radius of the cylinder $r_{\text {in }}$ and the inner radius of the dielectric $r_{\mathrm{d} i}$. The spacing of the rings is also a parameter and is defined by the length $s_{i}$ that denotes the position of each ring from the first ring $\left(s_{1}=0\right)$. We see that our modelling considers most of the geometric features of the real structure except of the tapering of the inner radius. At the borders of the geometry open boundary conditions are applied and the beam tunnel is modelled as an aperiodic structure of finite length.

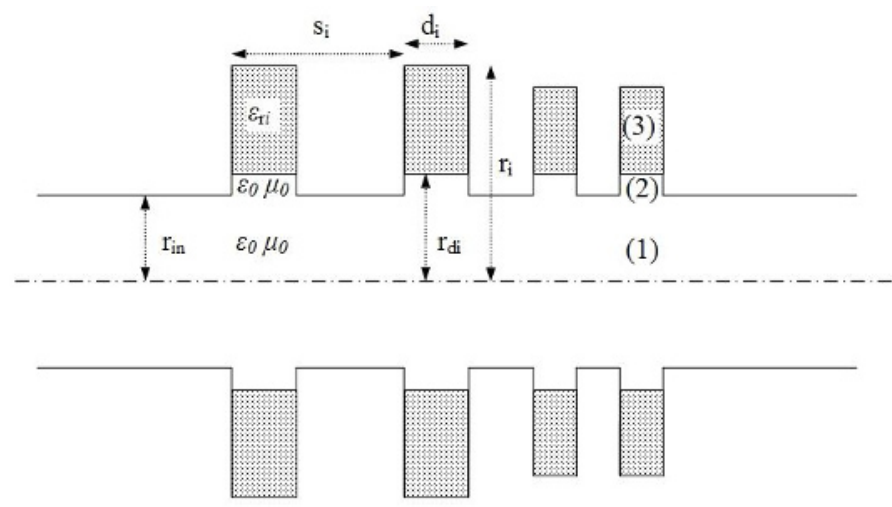

Fig. 1. Geometry of the modelled structure.

\subsection{Formulation of the problem}

We start the formulation of the problem by expressing the fields in the three regions in terms of electric and magnetic Hertz potentials that correspond to TM and TE modes. An assumed dependence of $\mathrm{e}^{+j \omega t}$ is suppressed throughout the rest of this paper. We start from region 3 (dielectric region) where the potentials are expressed as: 


$$
\begin{aligned}
& \boldsymbol{\Pi}_{e}^{(3)}=\hat{\mathbf{z}} \sum_{n=0}^{\infty} E_{n l} P_{n l}^{(3)}\left(k_{n l}^{(3)} r\right) e^{j m \varphi} \cos \left(\frac{n \pi}{d_{l}}\left(z-s_{l}\right)\right) \\
& \boldsymbol{\Pi}_{\mathbf{m}}^{(\mathbf{3})}=\hat{\mathbf{z}} \sum_{n=1}^{\infty} H_{n l} Q_{n l}^{(3)}\left(k_{n l}^{(3)} r\right) e^{j m \varphi} \sin \left(\frac{n \pi}{d_{l}}\left(z-s_{l}\right)\right)
\end{aligned}
$$

$E_{n l}, H_{n l}$ are the amplitude coefficients of TM and TE modes in the $l$-th ring with axial index $n, m$ is the azimuthal index, $k_{n l}^{(3)}=\sqrt{\omega^{2} \varepsilon_{r l} \varepsilon_{0} \mu_{0}-\left(n \pi / d_{l}\right)^{2}}$ is the transverse wavenumber in the dielectric and the transverse profile functions, in order to satisfy the boundary conditions at $r=r_{l}$, are defined as:

$$
\begin{aligned}
& P_{n l}^{(3)}\left(k_{n l}^{(3)} r\right)=J_{m}\left(k_{n l}^{(3)} r\right) N_{m}\left(k_{n l}^{(3)} r_{l}\right)-J_{m}\left(k_{n l}^{(3)} r_{l}\right) N_{m}\left(k_{n l}^{(3)} r\right) \\
& Q_{n l}^{(3)}\left(k_{n l}^{(3)} r\right)=J_{m}\left(k_{n l}^{(3)} r\right) N_{m}^{\prime}\left(k_{n l}^{(3)} r_{l}\right)-J_{m}^{\prime}\left(k_{n l}^{(3)} r_{l}\right) N_{m}\left(k_{n l}^{(3)} r\right)
\end{aligned}
$$

In the recess (region 2) the Hertz potentials have the following form:

$$
\begin{aligned}
& \boldsymbol{\Pi}_{\mathbf{e}}^{(2)}=\hat{\mathbf{z}} \sum_{n=0}^{\infty} P_{n l}^{(2)}\left(k_{n l}^{(2)} r\right) e^{j m \varphi} \cos \left(\frac{n \pi}{d_{l}}\left(z-s_{l}\right)\right) \\
& \boldsymbol{\Pi}_{\mathbf{m}}^{(2)}=\hat{\mathbf{z}} \sum_{n=1}^{\infty} Q_{n l}^{(2)}\left(k_{n l}^{(2)} r\right) e^{j m \varphi} \sin \left(\frac{n \pi}{d_{l}}\left(z-s_{l}\right)\right)
\end{aligned}
$$

where $k_{n l}^{(2)}=\sqrt{\omega^{2} \varepsilon_{0} \mu_{0}-\left(n \pi / d_{l}\right)^{2}}$ and $P_{n l}^{(2)}, Q_{n l}^{(2)}$ are defined as:

$$
\begin{aligned}
& P_{n l}^{(2)}\left(k_{n l}^{(2)} r\right)=\left(C_{1 e} E_{n l}+C_{1 h} H_{n l}\right) J_{m}\left(k_{n l}^{(2)} r\right)+\left(C_{2 e} E_{n l}+C_{2 h} H_{n l}\right) N_{m}\left(k_{n l}^{(2)} r\right) \\
& Q_{n l}^{(2)}\left(k_{n l}^{(2)} r\right)=\left(C_{3 e} E_{n l}+C_{3 h} H_{n l}\right) J_{m}\left(k_{n l}^{(2)} r\right)+\left(C_{4 e} E_{n l}+C_{4 h} H_{n l}\right) N_{m}\left(k_{n l}^{(2)} r\right)
\end{aligned}
$$

By applying the continuity of tangential electric and magnetic fields we can find the coefficients $C_{i e}, C_{i h},(i=1 \ldots 4)$ in analytic form. If the azimuthal mode index $m$ is 0 then $C_{1 h}, C_{2 h}, C_{3 e}, C_{4 e}=0$ and as a result TE and TM modes decouple.

In the hollow region (region 1), where the spectrum is continuous the Hertz potentials can be expressed as:

$$
\begin{aligned}
& \boldsymbol{\Pi}_{\mathbf{e}}^{\mathbf{s}}=\hat{\mathbf{z}} \int_{-\infty}^{+\infty} A_{e}(\zeta) J_{m}\left(k_{\perp} r\right) e^{j m \varphi} e^{j \zeta z} d \zeta \\
& \boldsymbol{\Pi}_{\mathbf{m}}^{\mathbf{s}}=\hat{\mathbf{z}} \int_{-\infty}^{+\infty} A_{m}(\zeta) J_{m}\left(k_{\perp} r\right) e^{j m \varphi} e^{j \zeta z} d \zeta
\end{aligned}
$$

where $k_{\perp}=\sqrt{\omega^{2} \varepsilon_{0} \mu_{0}-\zeta^{2}}$ and $A_{\mathrm{e}}(\zeta), A_{\mathrm{m}}(\zeta)$ are the Fourier transforms of the spatial dependence of the scattered TM and TE modes.

Finally, the excitation to our problem is assumed as an incident TE mode propagating from the left of the structure:

$$
\boldsymbol{\Pi}_{\mathbf{m}}^{\mathbf{s}}=\hat{\mathbf{z}} A J_{m}\left(k_{\perp}^{i n c} r\right) e^{j m \varphi} e^{-j \beta z}
$$

In order to match the boundary conditions at $r=r_{l}$ we proceed as in [3]. In this work we generalize the analytic results of [3] to apply also for general azimuthal index $m$ and for rings with different widths $d_{l}$. First the tangential magnetic fields are matched at the interface of the rings. Then we multiply with the eigenfunctions of each ring and we integrate over the ring's cross section. Two sets of infinite equations for each tangential magnetic field component are derived. Next we match the Fourier transforms of the tangential electric fields at the interface while also enforcing them to be zero elsewhere. By combining the equations and truncating higher order modes we get a linear system of $2 N+L$ equations for the mode coefficients $E_{n l}, H_{n l}$. Let $N_{l}$ to be the highest axial order of the modes we keep in the $l$-th ring. Then $N$ is the sum of $N_{l}$ for all rings and $L$ is the number of the rings. The $2 N+L$ equations (for $n=0,1, \ldots, N_{l}$ and $l=1,2, \ldots, L$ ) after combining the equations of continuity and some analytic manipulations are: 


$$
\begin{gathered}
{\left[\frac{n \pi}{d_{l}} m \frac{Q_{n l}^{(2)}\left(k_{n l}^{(2)} r_{i n}\right)}{r_{i n}}-k_{n l} \omega \varepsilon_{0} P_{n l}^{\prime}\left(k_{n l}^{(2)} r_{i n}\right)\right] \frac{d_{l}}{2}\left(1+\delta_{n 0}\right)+\sum_{v, \lambda} \frac{v \pi}{d_{\lambda}} \frac{m k_{v \lambda}^{(2)}}{r_{i n}} Q_{v \lambda}^{\prime}\left(\kappa_{v \lambda}^{(2)} r_{i n}\right) I_{1}(n, l, v, \lambda)} \\
-\sum_{v, \lambda} \frac{P_{v \lambda}^{(2)}\left(\kappa_{v \lambda}^{(2)} r_{i n}\right)}{r_{i n}^{2} \omega}\left[\left(\frac{v \pi}{d_{\lambda}}\right)^{2} \frac{m^{2}}{\mu_{0}} I_{1}(n, l, v, \lambda)-\left(k_{v \lambda}^{(2)}\right)^{2} \frac{m^{2}}{\mu_{0}} I_{3}(n, l, v, \lambda)+\left(\omega r_{i n} k_{v \lambda}^{(2)}\right)^{2} \varepsilon_{0} I_{4}(n, l, v, \lambda)\right] \\
=\frac{\beta^{2}}{r_{i n}} A m J_{m}\left(k_{\perp}^{i n c} r_{i n}\right) e^{-j \beta s_{l}} F_{n l}(\beta) \\
\left(k_{n l}^{(2)}\right)^{2} Q_{n l}^{(2)}\left(k_{n l}^{(2)} r_{i n}\right) \frac{d_{l}}{2}\left(1-\delta_{n 0}\right)+\sum_{v, \lambda} k_{v \lambda}^{(2)} \frac{n n \pi^{2}}{d_{l} d_{\lambda}} Q_{v \lambda}^{\prime}\left(\kappa_{v \lambda}^{(2)} r_{i n}\right) I_{2}(n, l, v, \lambda) \\
-\sum_{v, \lambda} \frac{m n \pi}{\omega \mu_{0} r_{i n} d_{l}} P_{v \lambda}^{(2)}\left(\kappa_{v \lambda}^{(2)} r_{i n}\right)\left[\left(\frac{v \pi}{d_{\lambda}}\right)^{2} I_{2}(n, l, v, \lambda)-\left(k_{v \lambda}^{(2)}\right)^{2} I_{1}(n, l, v, \lambda)\right] \\
=\left(k_{\perp}^{i n c}\right)^{2} A J_{m}\left(k_{\perp}^{i n c} r_{i n}\right) \frac{n \pi}{d_{l}} e^{-j \beta s_{l}} F_{n l}(\beta)
\end{gathered}
$$

In the above linear system of equations the unknown mode coefficients are present through the functions $P_{n l}^{(2)}, Q_{n l}^{(2)}$ and their derivatives. In general the solution is described by hybrid waves of both polarizations but for symmetric modes $(m=0)$ we can see that the two sets of equations decouple and the structure supports independent TE and TM modes.

From the procedure of matching the discrete spectrum of modes in the rings with the continuous spectrum of modes in the hollow region, the Fourier integrals $I_{i}(n, l, v, \lambda), i=1 \ldots 4$ arise:

$$
\begin{gathered}
I_{1}(n, l, v, \lambda)=\frac{1}{2 \pi} \int_{-\infty}^{+\infty} \frac{\zeta^{2} J_{m}\left(k_{\perp} r_{i n}\right)}{k_{\perp} J_{m}^{\prime}\left(k_{\perp} r_{i n}\right)} e^{j \zeta\left(s_{l}-s_{\lambda}\right)} F_{n l}(-\zeta) F_{v \lambda}(\zeta) d \zeta \\
I_{2}(n, l, v, \lambda)=\frac{1}{2 \pi} \int_{-\infty}^{+\infty} \frac{k_{\perp} J_{m}\left(k_{\perp} r_{i n}\right)}{J_{m}^{\prime}\left(k_{\perp} r_{i n}\right)} e^{j \zeta\left(s_{l}-s_{\lambda}\right)} F_{n l}(-\zeta) F_{v \lambda}(\zeta) d \zeta \\
I_{3}(n, l, v, \lambda)=\frac{1}{2 \pi} \int_{-\infty}^{+\infty} \frac{\zeta^{4} J_{m}\left(k_{\perp} r_{i n}\right)}{k_{\perp}^{3} J_{m}^{\prime}\left(k_{\perp} r_{i n}\right)} e^{j \zeta\left(s_{l}-s_{\lambda}\right)} F_{n l}(-\zeta) F_{v \lambda}(\zeta) d \zeta \\
I_{4}(n, l, v, \lambda)=\frac{1}{2 \pi} \int_{-\infty}^{+\infty} \frac{\zeta^{2} J_{m}^{\prime}\left(k_{\perp} r_{i n}\right)}{k_{\perp} J_{m}\left(k_{\perp} r_{i n}\right)} e^{j \zeta\left(s_{l}-s_{\lambda}\right)} F_{n l}(-\zeta) F_{v \lambda}(\zeta) d \zeta \\
F_{n l}(\zeta)=\frac{1-(-1)^{n} e^{-j \zeta d_{l}}}{\left(n \pi / d_{l}\right)^{2}-\zeta^{2}}
\end{gathered}
$$

The Fourier integrals can be evaluated analytically in the form of rapidly convergent series by means of contour integration and residue theory. The poles of the integrands come from the zeros of Bessel function $\left(\zeta_{m p}=\sqrt{\omega^{2} \varepsilon_{0} \mu_{0}-\chi_{m p}^{2}}\right)$ or its derivative $\left(\zeta_{m p}^{\prime}=\sqrt{\omega^{2} \varepsilon_{0} \mu_{0}-\chi_{m p}^{\prime 2}}\right)$ where $\chi_{m p}$ and $\chi_{m p}^{\prime}$ are respectively the roots of Bessel function and its derivative. We introduce an infinitesimal imaginary part to the frequency in order to move the poles $\zeta_{m p}, \zeta_{m p}^{\prime}$ away from the real axis. There are also poles at $\zeta= \pm n \pi / d_{l}$ that are accounted for by their principal value. The path of integration and the poles are shown in Figure 2. The path can be closed in the upper-half or lower-half of the complex plane depending on the relative position between the $l$-th and $\lambda$-th ring. Application of Cauchy theorem gives us in closed form the Fourier integrals. Therefore the elements of the matrix of the linear system can be computed efficiently. 


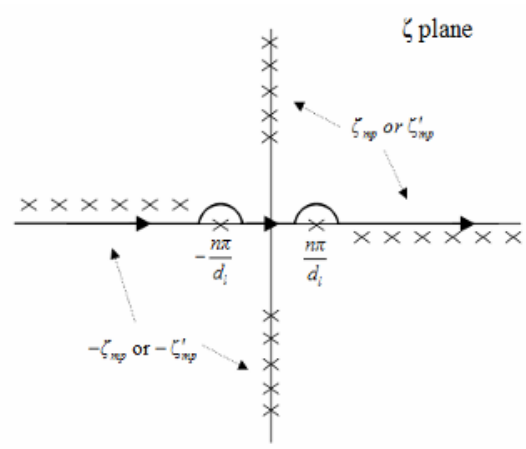

Fig. 2. Integration path on complex plane

\subsection{Computer Simulation}

The code developed takes as input the geometry of the beam tunnel and the parameters of the incident modes. As we have presented, the matrix of the linear system can be filled efficiently. The inversion of the matrix is rapid and trivial because the size of the matrix for real beam-tunnel geometries is generally small. From the mode coefficients, the absorbed power can be directly computed.

The scattered field at the hollow region can be analytically evaluated too. We can express the functions $A_{\mathrm{e}}(\zeta)$ and $A_{\mathrm{m}}(\zeta)$ from the mode coefficients. By evaluating the Fourier integrals that come up by the same method as $I_{i}(n, l, v, \lambda)$, we can have analytical formulas about the fields in the hollow region. Therefore the field is known in every region and can be computed efficiently.

\section{Results and discussion}

\subsection{Results}

We present in Figure 3 the azimuthal electric field for a geometry resembling the beam tunnel of the W7-X $140 \mathrm{GHz}$ gyrotron [4]. The parameters of the simulation are referred to in Table 1. We can see from the figure that the field penetrates only slightly the dielectric region. Therefore the absorption is minor as the field is reflected to a large extent by the dielectric. In order for the field to penetrate the dielectric and the absorption to be significant there should be a good matching between the vacuum region and the dielectric. The matching depends on the dielectric constant and also on the geometric properties of the rings. Analytic formulas describing the matching can be derived from our model.

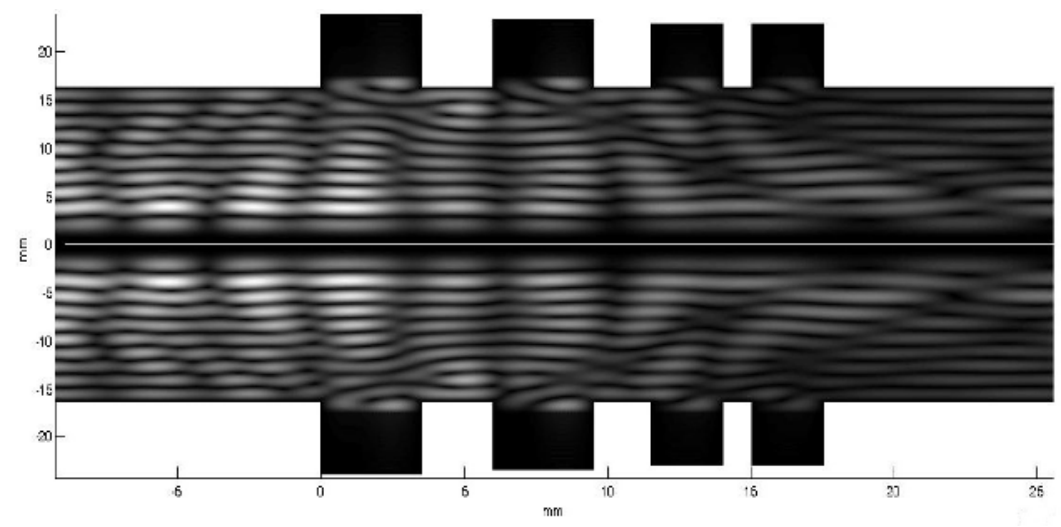

Fig. 3: The magnitude of electric field $E_{\varphi}$ for the parameters of table I 
Table 1. Parameters for the simulation of Figure 3.

\begin{tabular}{|c|c|c|c|c|c|c|}
\hline Incident modes & Frequency & Rings width & $\begin{array}{c}\text { Inner } \\
\text { radius }\end{array}$ & $r_{i}$ & $\begin{array}{c}\varepsilon_{\mathrm{r} i} \\
i=1,2,3,4\end{array}$ & $\begin{array}{c}r_{\mathrm{d} i} \\
\mathrm{i}=1,2,3,4\end{array}$ \\
\hline $\begin{array}{c}\text { TE } 5,10 \\
\text { TM 5,9 }\end{array}$ & $116 \mathrm{GHz}$ & $\begin{array}{c}d_{1,2}=3.5 \mathrm{~mm} \\
d_{3,4}=2.5 \mathrm{~mm}\end{array}$ & $16.2 \mathrm{~mm}$ & $\begin{array}{c}r_{l}=23.8 \mathrm{~mm} \\
r_{2}=23.3 \mathrm{~mm} \\
r_{3,4}=22.8 \mathrm{~mm}\end{array}$ & $25-1 \mathrm{j}$ & $17.5 \mathrm{~mm}$ \\
\hline
\end{tabular}

\subsection{Possible extensions}

Since our new model treats the beam tunnel structure as finite, the foundation has been set for further enhancements of the model, to consider even more realistic structures. First of all, instead of external excitation modes, we can find the eigenmodes that the structure supports as an open resonator. If we remove the incident field that defines the right hand side of the linear system and search the complex plane for frequencies that make zero the determinant of the matrix, we get the complex eigenfrequencies of the cold structure. The imaginary part of the complex eigenfrequencies describes the dielectric losses as well as the diffraction losses. As the longitudinal field profile of open resonators is exponentially increasing at the ends, the Fourier transform classically does not exist. In order to account for the proper exponentially increasing solutions the contour path of the inverse Fourier transform should be deformed to contain the proper poles.

If the parasitic interaction is of a gyrotron type then the frequency will be near the eigenfrequency of the cold structure. In order to account also for the Gyro-BWO interaction the beam-wave interaction should be considered in a self-consistent manner. A varying magnetostatic field can be also modelled as the structure is now finite and not periodic.

A more realistic geometry should also consider the tapering of the inner radius $r_{\text {in }}$ or more general boundary conditions instead of open or periodic. Finally the azimuthal corrugations on the metal rings could also be modelled by enhancing the model with all azimuthal harmonics and by applying the matching of the modes at the interfaces.

\section{Acknowledgement}

This work was supported by and performed for the Association EURATOM - Hellenic Republic, funded by the EURATOM Fusion Programme and the Secretariat for Research and Technology, Greece. The contents of this paper are the sole responsibility of the authors and do not necessarily represent the views of the European Commission or its Services.

\section{References}

1. I. G. Tigelis, J. L. Vomvoridis, S. Tzima, IEEE Trans. Plasma Sci., 26, 922-930, (1998)

2. G. P. Anastasiou, G. P. Latsas, I. G. Tigelis, M. Dehler, P. Queffelec, N. F. Dasyras, IEEE Trans. Plasma Sci., 32, 1310-1317, (2004)

3. H.S. Lee, H.J. Eom, IEEE Trans. Microwave Theory Tech., 49, 315-320, (2001)

4. G. Dammertz et al, IEEE Trans. Plasma Sci., 30, 808-818, (2002) 